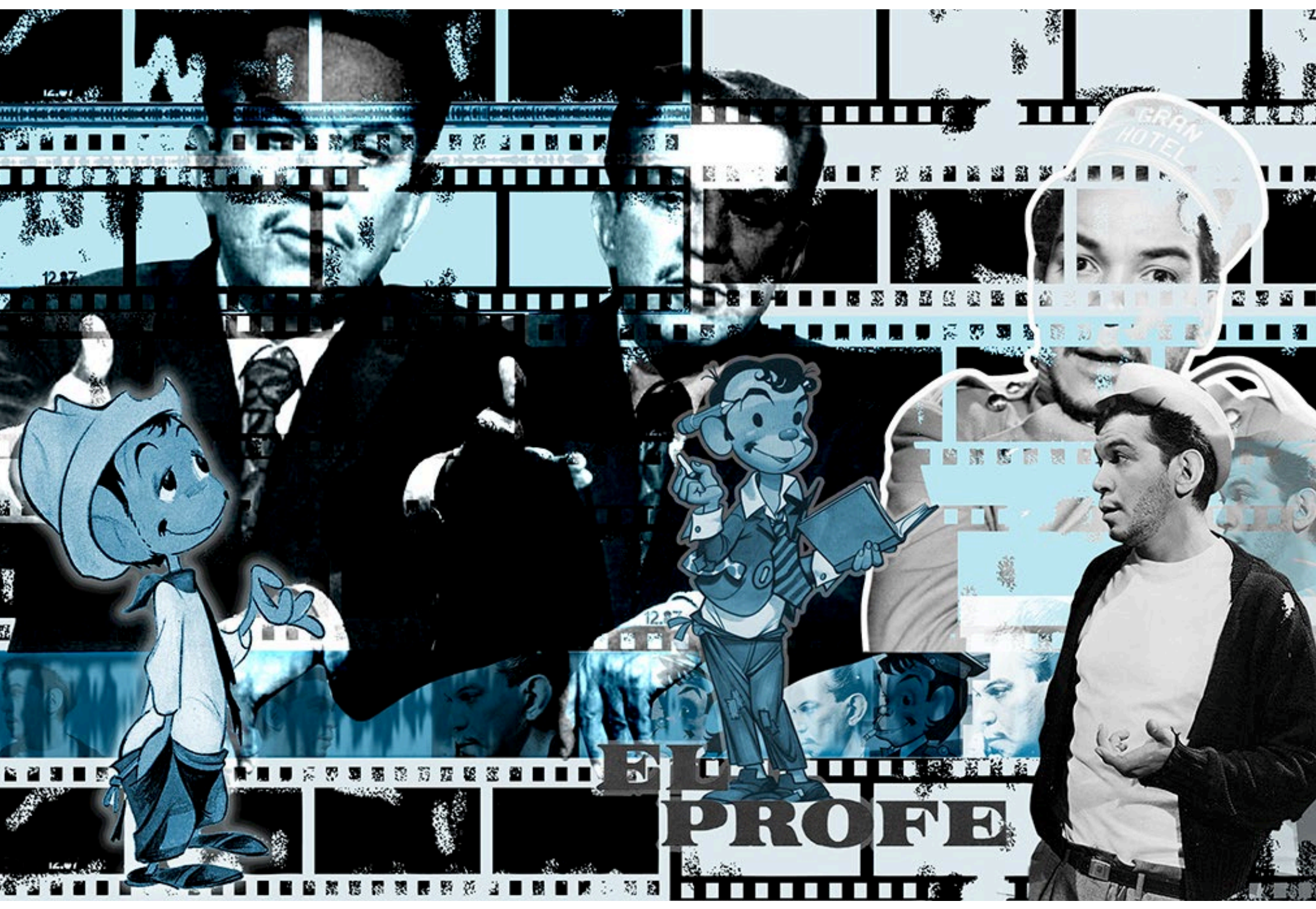

\title{
Formación humana, interculturalidad e inclusión desde el cine clásico de Cantinflas
}

Human formation, interculturality and inclussion from the Cantiflas classic cinema

Formação humana, interculturalidade e inclusão do cinema clássico de Cantinflas José Eriberto Cifuentes Medina 


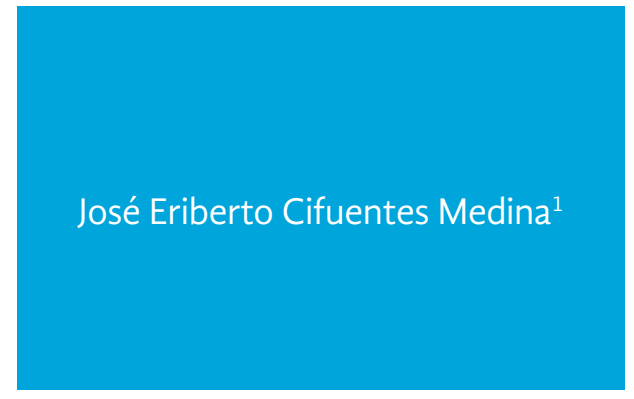

Doctorando en Educación, Universidad Baja California, México; Magister en Educación, Especialista en Educación con Énfasis en Evaluación Educativa; Licenciado en Teología, Licenciado en Filosofía y Educación Religiosa, Universidad Santo Tomás. Docente, Universidad Pedagógica y Tecnológica de Colombia; correo electrónico: joseeriberto.cifuentes@uptc.edu.co

Fecha de recepción: 7 de junio de 2018 / Fecha de aprobación: 26 de septiembre de 2018

\section{Resumen}

La investigación expuesta en el presente artículo se centró en el análisis de la formación humana en estudiantes de educación a distancia, con la incorporación de la asignatura de "Humanidades, el cine de Cantinflas" al corpus académico. Se integraron veinte películas como objeto de estudio, buscando analizarlas desde la perspectiva de su aporte significativo a la formación humana, proyectando un vínculo con la eficiencia en el desempeño laboral y profesional. Se encontró que el cine puede ser fácilmente introducido en los contenidos del proceso de enseñanzaaprendizaje y que implica una reflexión enriquecedora de la educación. Se dio una relación entre la formación humana y el cine clásico de Mario Moreno en el proceso formativo de los tecnólogos que fortalece el perfil profesional.

Palabras clave: Cine, educación a distancia, estudiantes, formación humana.

\section{Abstract}

The research presented in this article focused on the analysis of the human formation in online education students, with the incorporation of the subject "Humanities, the Cantinflas' Cinema" to the academic corpus. There were integrated twenty films as an object of study, seeking to analyze them from the perspective of their significant contribution to human formation, projecting a link with efficiency in professional performance. It was found that the cinema can be easily introduced in the contents of the teaching-learning process and that it implies an enriching reflection about education. It was developed a relationship between human formation and the Mario Moreno classic cinema in the formative process of the technologists which strengthens the professional profile.

Keywords: Cinema, online education, students, human formation.

\section{Resumo}

A pesquisa apresentada no presente artigo focado na análise do formação humana em estudantes de educação a distância, com a incorporaçãodotema"Humanidades, cinema de Cantinflas "para o corpus acadêmico. Ele eles integraram vinte filmes como um objeto de estudo, buscando analisá-los a partir de a perspectiva da sua contribuição significativa à formação humana, projetando um link com eficiência de desempenho trabalho e profissional Verificou-se que o cinema pode ser facilmente introduzido em o conteúdo do processo de ensinoaprendizagem e isso implica uma reflexão educação enriquecedora Aconteceu uma relação entre a formação humana e o cinema clássico de Mario Moreno no processo formativo dos tecnólogos que Fortalece o perfil profissional.

Palavras-chave: Cinema, educação a distância, alunos, formação humana. 


\section{Introducción}

$$
\mathrm{L}
$$

a propuesta de investigación que se presenta a continuación se trabaja desde la asignatura de Humanidades, contemplada - en el plan de estudios y parte del área general, junto a: Competencias comunicativas y métodos de estudio, Cultura política y ética. La asignatura es considerada teórica y se orienta en los programas de: Gestión en Salud y Regencia de Farmacia; los cuales fueron objeto de estudio, en la primera se orienta en primer semestre y en la segunda en tercero.

El trabajo se realizó desde el ejercicio de la docencia y la libertad de cátedra, pero cumpliendo con los principios básicos de los micro currículos; se incorporó el cine clásico, desde la obra de Mario Fortino Alfonso Moreno Reyes, más conocido como "Cantinflas", como una experiencia práctica, pues se considera que puede contribuir a la formación humana de los tecnólogos en Gestión de la Salud y Regencia de Farmacia, considerando que ellos, como agentes destinados a prestar sus servicios en el área de la salud, deben permear su vida de valores morales, éticos y sociales idóneos, que contemplen al ser humano integral, de tal forma que no pierdan jamás, justamente, su condición humana.

La investigación se encuentra enmarcada en un trabajo de carácter práctico, por lo que ha implicado que los estudiantes realicen un análisis de la formación humana, la inclusión, las humanidades, la interculturalidad y la globalización, todo, desde el cine de Cantinflas, desglosando los principios implícitos en la obra para lograr una formación integral e idónea como futuros agentes de la salud.

Se trata de una experiencia de aula en proceso de construcción, con avances en el campo de acción, desde el proceso de enseñanzaaprendizaje, enfocada en la edificación sólida de una formación humana, con fundamentos claros de inclusión e interculturalidad, para lograr establecer un desarrollo social del estudiante, de tal forma que sea justo, equilibrado y que cuente con bases fuertes de valores, cuyo origen sea la familia y su desarrollo se dé en la escuela, integrándose al sistema educativo, pues la formación en valores es deber de todos.

\section{Generalidades teóricas}

En el desarrollo teórico de la investigación se parte de tres tópicos de profundización: Formación humana, inclusión e interculturalidad; los cuales contribuyen a fortalecer la experiencia de aula en el proceso de enseñanza-aprendizaje de las humanidades desde la perspectiva del cine clásico de Cantinflas.

\section{La formación humana}

El humanismo tiene su fuerza en la posibilidad de rescatar y preservar la libertad del ser humano y los Derechos Humanos, como elementos preciados en el ser y esencia de la sociedad; por ello:
Una de las tareas del humanismo es convertir al hombre en un ser humano a plenitud. Adquiere mayor significación en nuestro tiempo que experimenta una gran deshumanización [...] La naturaleza de lo humanístico demanda un tratamiento en el cual debe ir comprometida la formación integral del hombre, orientada en la interdisciplinariedad pertinente y en el diálogo entre disciplinas de complementación recíproca (Recio, 1998, pp. 53-54).

Es importante formar a los profesionales en la esencia del desarrollo, como seres humanos competentes en el saber; entendido este último como saber-saber, saber-ser y saber-hacer, cuyo centro vital sería el de ser, es decir, un profesional especializado en el saber humanístico. El humanismo ha de contribuir a la transformación humana del propio espíritu, que proyecte la esencia de tal plenitud de vida a los demás.

En cuanto a la esencia de las competencias, se hace necesario que el estudiante sea un ser humano integral en todo el sentido de la palabra, capaz de conocerse y descubrirse a sí mismo; tal vez así podrá ser mejor persona y agente generador de una transformación social, comprometido con el análisis y la valoración de la realidad, entre tanto:

\footnotetext{
Las humanidades están llamadas a cultivar los valores humanos, es necesario que tomen como propia la causa del hombre actual y busquen un nuevo humanismo, esta sería una estrategia para salvar el abismo que se da entre las ciencias y las humanidades. En tal sentido, las humanidades deben aceptar este reto, para que sean fieles al hombre e intérpretes de sus auténticas necesidades y problemas (Recio, 1998, p. 60).
}

En el nuevo humanismo, las humanidades deben renovarse y humanizar los programas universitarios, con más razón aquellos de carácter tecnológico. En el ejercicio de la docencia se trata de formar estudiantes como hombres capaces de "comprender el contexto en que se desenvuelve su devenir y su quehacer, pero también capaz de proponer nuevas opciones para mejorar la educación y desarrollo de actitudes que lo comprometan con dicha acción" (Recio, 1998, p. 61). Es una forma de ampliar el radio de acción educativo, incluso fuera del aula de clase, para que transcienda en la familia y el trabajo, aspirando a una Colombia mejor, más justa, más humana, más solidaria y en paz. 
La historia juega un papel importante en la vida humana y en la sociedad, por lo que es importante saber que lo fundamental es "poner al alumno en contacto con la apasionante aventura humana, para que descubra que en el hombre hay más cosas dignas de admiración que de desprecio" (Echeverry, 1999, p. 59). Para el hombre no es fácil afrontar su propia realidad en su devenir histórico, pero le es necesario acudir a su condición humana para:

[...] que al tiempo que descubre, se familiarice con las distintas técnicas y recursos que, cuando los adquiera, le permitan su autónomo investigar, estudiar, analizar, ponderar, criticar, relativizar, no para la escuela sino para la vida, de modo que sienta orgulloso de ir conquistando herramientas que le permitan aprender haciendo, que le permitan aprender a aprender, que le den la certeza de que cuenta con los medios para afrontar la única tarea de su existencia: desarrollar al máximo sus posibilidades de ser fiel a sí mismo (Echeverry, 1999, pp. 59-60).

El ser humano necesita de la humanización de su condición, pues ésta se da en la realidad de comprender que es humano y de que, por ende, necesita formar su humanidad; una paradoja que al ser entendida le permite ver la responsabilidad de su papel en la sociedad. El cine clásico es un gran instrumento para la formación humana, pues permite preguntar el por qué de la vida e incluso intentar responder dichos interrogantes. Como espectadores, es posible experimentar una conmoción profunda y purificadora, y tomar conciencia de los mejores aspectos de nuestro ser, en definitiva, de nuestro sentido.

La humanización es necesaria en todos los campos profesionales y en todos los contextos donde se desenvuelve el ser humano entrando en relación con sus semejantes; esto es muy importante en la salud, escenario que ofrece múltiples ejemplos de negligencia por parte de quienes se desempeñan en las diferentes dependencias, claro, considerando las excepciones del caso, pues siempre hay quienes tienen un mayor desarrollo humanista.

\section{Inclusión pedagógica}

En medio de las diversidades humanas, pues independientemente de la teoría de origen del hombre (creacionista, evolucionista, etc.), una característica genérica es nuestra diversidad en esencia y existencia; a pesar de las similitudes genéticas, cada persona es diferente:

En toda sociedad coexisten las diversidades humanas. En cada situación concreta las mujeres y los hombres desean disfrutar los derechos económicos, sociales y culturales, especialmente el derecho a la educación, pues saben que quienes no han tenido acceso a la escuela, si bien pueden estar presentes en el escenario social, discutir asuntos comunes y votar en cualquier proceso democrático [no pueden disfrutarlo a plenitud] (Munévar, 2008).

Un factor fundamental en la sociedad y en la educación es el de contribuir a que los humanos, a pesar de su diversidad, puedan convivir como especie y entre generaciones. El ser humano ha de comprender que existe la diversidad y que en este espacio puede excluir o incluir a sus semejantes: "este debate ha dado origen a la inclusión en la educación, una iniciativa que interroga tanto a la políticas públicas en abstracto como a las prácticas sociales en concreto" (Munévar, 2008). Así, las instituciones educativas tienen la necesidad de educar para la inclusión social, para comprender las diversidades de nuestra sociedad.

Se trata de favorecer el ingreso, permanencia y formación de los estudiantes en las instituciones educativas, para que quienes se encuentren en situación de diferencia social, económica, geográfica, lingüística, cultural o individual, puedan disponer de las condiciones institucionales para la inclusión en el desarrollo educativo; por ello es necesario introducir prácticas inclusivas en los agentes educativos, evitando:

Lenguajes excluyentes, avanzando con las repercusiones institucionales [que] se concretan con las necesidades e intereses de cada estudiante, el alcance de los enfoques educativos, la revisión de buenas prácticas escolares y la re-significación de los componentes del proceso educativo; esto es, de las complejas relaciones entre escuela e inclusión (Munevar, 2008).

Es pertinente trabajar los dos contextos de manera paralela, ambientes de exclusión y de inclusión en la escuela, para comprender las dos direcciones y evitar la exclusión a costa de lo que sea, más cuando se trata de educación. La inclusión ha de ser la bandera de los seres humanos, el respeto por la diversidad, cumpliendo los deberes y derechos como normas básicas de la sociedad.

En las instituciones educativas la inclusión "supone la definición de múltiples estrategias para abordar y resolver las resistencias emergentes; define el ejercicio del derecho a asistir a la escuela como el estar presente en un escenario en que se puedan vivir las experiencias académicas" (Munévar, 2008). Todas las estrategias de los docentes para evitar la marginación, la expulsión, exclusión o desarraigo de los estudiantes, han de ser tenidas en cuenta, pues todo lo que favorece la inclusión en el aula fomenta el desarrollo de la formación humana. 
El reto de la educación inclusiva es eliminar todo acto de discriminación por discapacidad, cultura, género, religión o cualquier otra diferencia, para que los estudiantes dispongan, en la misma medida, de "las oportunidades para acceder a un currículo culturalmente valioso a tiempo completo, como miembros de una aula acorde con su edad, por incentivar los principios de participación (...) la protección de derechos y el cumplimiento de deberes” (Munévar, 2008). En tal sentido, el cine clásico puede contribuir a la formación humana de los estudiantes, pues desde diferentes películas promueve la inclusión social.

\section{La interculturalidad}

El desarrollo de la interculturalidad en la sociedad es un asunto complejo, pues favorece a unos y desfavorece a otros, ya que algunas personas podrían considerar el proceso como usurpación de su propia cultura; además, puede pasar de inter a multi-culturalidad, es decir, perdiendo principios de identidad propia que se mezclan con principios de la diversidad. Algunos ven este fenómeno como hecho favorable para los tiempos de la globalización, pero muchos sienten que se pierde un capital preservado por generaciones; así:

La interculturalidad reconoce las redes de relaciones sociales que se han constituido y construido a partir de la historia y la dinámica que se ha generado en el encuentro o desencuentro con el otro, de la interacción, las contradicciones, el intercambio, la sociabilidad y la reciprocidad, de los conflictos, las resistencias, las rupturas, los símbolos y el poder (González, 2005).

La interculturalidad puede verse como un proceso que pasa, de un diálogo entre las unidades de una sola cultura, a una conversación entre varias civilizaciones, o mejor, un movimiento que entrelaza a más de una cultura; por tanto, la escuela es un espacio en el que "el reconocimiento de la interculturalidad o de la diversidad cultural no debe ser un debate que se quede en lo estrictamente académico, sino que debe permear a la sociedad y en particular a la escuela como espacio de socialización" (González, 2005). Las cosas no son más fáciles cuando la escuela se define desde la homogeneidad, la uniformidad, por ello:

La interculturalidad es el producto de la relación que se establece entre los diferentes grupos étnicos de una región y, a su vez, la articulación de estos grupos étnicos con la sociedad hegemónica nacional. La interculturalidad toma como punto de partida los elementos propios de la cultura del grupo étnico local que se proyectan en relación con los otros, permitiendo la apropiación de ciertos elementos que para el grupo étnico local armonicen con su cultura o le sirvan para interactuar con otros grupos que entran en contacto con él (Forbes, et al., 1992).

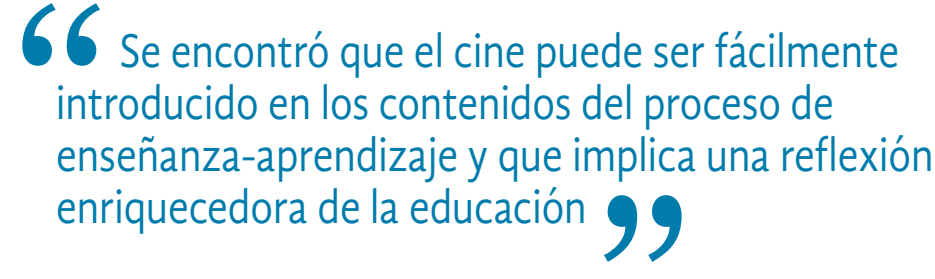

Una sociedad intercultural es la que en su interior comporta varias culturas; un aula de clase puede ser intercultural cuando brilla el respeto por todos en igualdad de condiciones, agregando la participación de los grupos minoritarios y el respeto por sus tradiciones, costumbres, lengua, religión, etc. En tal contexto, los valores son necesarios para el desarrollo humano en medio de la diversidad, por ello se debe rescatar la cultura de los grupos minoritarios, pues hacen parte de la entraña de la sociedad, mucho más en las instituciones educativas, pues:

La educación ha sido un factor de desadaptación social y de violencia simbólica en el cual el ciudadano de un grupo minoritario termina por vivir apenado de su cultura y lengua ante su propia cultura y lengua, ante propios y extraños, sin saber de dónde viene ni para donde va. ¡Viva la santa aculturación! (Forbes, et al., 1992).

El cine clásico de Cantinflas aporta desde a la formación del ser humano a la inclusión social y pedagógica en el contexto educativo, pues asume a la persona como ser para ser respetado en la sociedad, que ocupa un lugar y lo merece; tal como la interculturalidad, su cine es muy respetuoso de los principios de cada cultura y en cada contexto social. Junto a ello, vale la pena reconocer otros factores que se insertan en el proceso de inter y multiculturalidad, como la importancia de la globalización, que es:

[...] tanto un conjunto de procesos de homogenización, como fraccionamiento articulado del mundo, que reordenan las diferencias y las desigualdades sin suprimirlas. O sea que estamos identificando una doble agenda de la globalización: por una parte, integra y comunica; por otra, segrega y dispersa (García, 1999).

La globalización contiene elementos que contribuyen a "borrar las diferencias ( $\mathrm{y}$ a) reordenarlas a fin de producir nuevas fronteras, menos ligadas a los territorios que a la distribución desigual en los mercados" (Forbes, et al., 1992). No solo se crean nuevas fronteras en el trabajo y el consumo: aumentan la eficacia al desconectar a quienes podrían organizarse para atravesarlas o derrumbarlas. 


\section{Metodología de la revisión}

El trabajo se apoya en el enfoque cualitativo, mientras que el tipo de investigación corresponde al método descriptivo (Castillo, 2004), asignando una población y muestra acordes con el contexto del proyecto. Al emplear el enfoque cualitativo, es importante reconocer que éste implica:

Descripciones detalladas de situaciones, eventos, personas, interacciones, conductas observadas y sus manifestaciones. El investigador cualitativo utiliza técnicas para recolectar datos, como la observación no estructurada, entrevistas abiertas, revisión de documentos, discusión en grupo, evaluación de experiencias personales (p. 9). [Se considera un enfoque abierto y] [...] en la mayoría de estudios cualitativos no se prueban hipótesis [...] se basa en métodos de recolección de datos no estandarizados ni completamente predeterminados [...] y se fundamenta en una perspectiva interpretativa, centrada en el entendimiento del significado de las acciones de seres vivos, sobre todo de los humanos y sus instituciones (Hernández, 2010).

Por otra parte, el tipo de investigación aplicado fue el descriptivo, el cual permite "registrar las características, las conductas y demás factores de hechos y fenómenos (...) busca fundamentalmente describir, pues su objetivo no es comprobar explicaciones, ni hacer predicciones" (Castillo, 2004). La experiencia se desarrolló en la materia de Humanidades, particularmente en las carreras de Tecnología en Regencia de Farmacia, tercer semestre, y Gestión en Salud, primer semestre, con una población total de 82 estudiantes de los dos programas. De dicha población se tomó un $50 \%$ como muestra, especialmente de las ciudades de Tunja, Duitama y Sogamoso, y de la carrera de Regencia en Farmacia

\section{Etapas del desarrollo}

El proyecto siguió varias etapas, algunas ya se han abordado y otras se siguen trabajando: la primera, de diagnóstico, consistió en elegir una película y desarrollar cuatro partes, para luego socializar en el aula y retroalimentar el trabajo académico-investigativo; la segunda, trabajo de campo, donde se aplicó una encuesta a los estudiantes de manera que, luego de realizada la actividad, se diera paso a la investigación y su aplicabilidad en la vida y la academia, desde la idea de que el cine de Cantinflas contribuye a la humanización de los servicios de la salud.

\section{Resultados y discusión}

\section{Primera etapa}

Proceso de diagnóstico que consiste en elegir una película y desarrollar cuatro puntos para luego socializar en el aula y retroalimentar. Se busca identificar los elementos necesarios para impulsar la formación humana de agentes en el área de la salud. A continuación se presenta una muestra del trabajo:

1. Luego de ver la película, sintetice a través de un dibujo o collage: Personajes principales; personajes secundarios y su relación con la interculturalidad, inclusión y las personas.

2. Elabore un texto de mínimo diez líneas con una reflexión exponiendo los aportes de la película a la educación y a la pedagogía para la inclusión.

3. ¿Cuáles son los aportes de Mario Moreno "Cantinflas" a: las Humanidades; la formación humana; la interculturalidad y la inclusión en la época de la globalización?

4. Elabore un organizador gráfico para presentar la síntesis de la película y su relación con la formación humana, la interculturalidad y la inclusión-

Figura 1. Exposición de trabajos

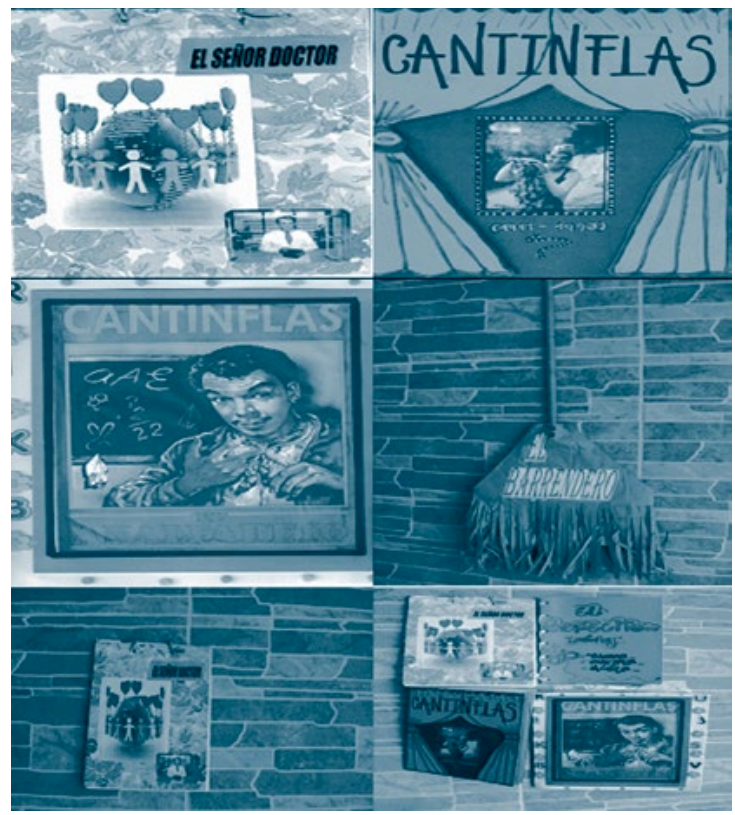

Nota. Fuente: estudiantes de Humanidades 
Cada una de las veinte películas siguió los cuatro puntos de trabajo académico-investigativo y su desarrollo da cuenta de la creatividad de todos los estudiantes.

\section{Segunda etapa}

Corresponde al trabajo de campo, en este momento se aplicó una encuesta a los estudiantes de manera que, luego de realizar una actividad, investigaran y buscaran formas de aplicar lo aprendido a la vida y la academia; cuenta con doce preguntas que luego fueron analizadas; todo, a partir de una encuesta técnica, la cual permite recolectar datos de los individuos de una población e identificar sus opiniones, apreciaciones y puntos de vista. Aquí se aplicó una encuesta semiestructurada a ochenta y dos estudiantes de los programas de Regencia de Farmacia y Gestión en Salud de los CREAD de Tunja, Duitama y Sogamoso; participaron sesenta y ocho mujeres y catorce hombres de los tres CREAD.

Figura 2. Generalidades por género

\begin{tabular}{|c|c|c|c|c|c|}
\hline Opción & Estudiantes & Porcentaje & \multirow{2}{*}{\multicolumn{3}{|c|}{$\begin{array}{l}\text { Encuestados por género } \\
80 \\
60\end{array}$}} \\
\hline Hombres & 14 & $17,07 \%$ & & & \\
\hline Mujeres & 68 & $85,93 \%$ & 40 & & \\
\hline Total & 72 & $100 \%$ & —Series 1 & $\begin{array}{c}\text { Hombres } \\
14\end{array}$ & $\begin{array}{c}\text { Mujeres } \\
68\end{array}$ \\
\hline
\end{tabular}

Nota. Fuente: información del instrumento aplicado

El estudio acude a dos de los programas adscritos a la escuela de Ciencias Administrativas y Económicas, asociados al área de la Salud: Regencia de Farmacia y Gestión en Salud. La población con mayor participación fue la femenina. Los dos programas son de pregrado en el nivel de tecnología, cuentan con un desarrollo normal de currículo en seis semestres y el estudio acudió a estudiantes que cursaban primer y tercer semestre al terminar II-2013 y en I-2014.

Figura 3. Estudiantes encuestados

\begin{tabular}{|c|c|c|c|c|c|}
\hline Opción & Estudiantes & Porcentaje & \multirow{2}{*}{\multicolumn{3}{|c|}{ Estudiantes por semestre }} \\
\hline I semestre & 38 & $46,3 \%$ & & & \\
\hline III semestre & 44 & $53,7 \%$ & 3 & I semestre & III semestre \\
\hline Total & 83 & $100 \%$ & - Series 1 & 38 & 44 \\
\hline
\end{tabular}

En la facultad de Estudios a Distancia funcionan alrededor de 23 Centros Regionales de Educación a Distancia (CREAD), para el estudio se tomaron estudiantes de Tunja, Duitama y Sogamoso.
Figura 4. CREAD que participaron de la propuesta

\begin{tabular}{|c|c|c|c|c|c|c|}
\hline Cread & Estudiantes & Porcentaje & \multirow{3}{*}{\multicolumn{4}{|c|}{ Encuestados por Cread }} \\
\hline Tunja & 42 & $51,20 \%$ & & & & \\
\hline Duitama & 22 & $26,80 \%$ & & & & \\
\hline \multirow{2}{*}{ Sogamoso } & \multirow{2}{*}{18} & \multirow{2}{*}{$22,00 \%$} & & Tunja & Duitama & Sogamoso \\
\hline & & & - Series 1 & 42 & 22 & 18 \\
\hline Total & 82 & $100 \%$ & & & & \\
\hline
\end{tabular}

Nota. Fuente: información del instrumento aplicado

El CREAD con mayor concentración de estudiantes es el de Tunja, pero se acudió a tres de ellos para tomar una muestra significativa, reconociendo que, por lo general, los estudiantes proceden de municipios aledaños a cada entidad. Se diseñó una encuesta de doce preguntas, de las cuales ocho fueron estructuradas, con posibilidad para justificar la respuesta, y cuatro para captar la opinión abierta de los estudiantes. La primera fue: ¿Considera importante introducir el cine clásico en los contenidos temáticos de la asignatura de Humanidades?

Figura 5. Cine clásico en los contenidos de Humanidades

\begin{tabular}{|c|c|c|c|c|c|}
\hline Opción & Estudiantes & Porcentaje & \multirow{2}{*}{\multicolumn{3}{|c|}{$\begin{array}{l}\text { Cine clásico en los contenidos } \\
100 \\
50\end{array}$}} \\
\hline Sí & 80 & $97,6 \%$ & & & \\
\hline No & 2 & $2,4 \%$ & & $\mathrm{Si}$ & No \\
\hline Total & 82 & $100,0 \%$ & - Series 1 & 80 & 2 \\
\hline
\end{tabular}

Nota. Fuente: información del instrumento

Es pertinente comenzar por destacar las dos respuestas que no vieron pertinente la inclusión del cine clásico en la asignatura de Humanidades, la primera se centró en el hecho de que jamás le parecieron interesantes las películas de Cantinflas; la segunda señaló que sería más adecuado hablar de cine en humanidades, no solo de aquél centrado en un personaje. Sin embargo, pese a estas dos expresiones, hubo una mayoría de respuestas positivas frente a la inclusión del cine clásico; dentro de ellas, los estudiantes manifestaron que:

Permite aprender y comprender los valores de una manera práctica y brinda elementos para la formación como persona en el desarrollo de la sociedad y de una profesión.

El cine de "Cantinflas" ayuda a dar sentido a un mundo sin sentido; a través de su forma jovial y agradable de actuar deja muchas enseñanzas para la vida.

Las películas contribuyen a enfrentar cada situación de la vida desde la profesión, a manejar el público como en la película de $\mathrm{Su}$ excelencia. 
Las películas promueven la calidad de vida como seres humanos y la capacidad de resolver problemas cotidianos con agilidad, también contribuyen a que, desde las empresas de la salud se dé una excelente atención a clientes y pacientes.

La segunda pregunta fue: ¿Puede el cine clásico de Mario Moreno aportar a la formación humana de un tecnólogo?

Figura 6. Aportes del cine clásico a la formación humana

\begin{tabular}{|c|c|c|c|c|c|}
\hline Opción & Estudiantes & Porcentaje & \multirow{2}{*}{\multicolumn{3}{|c|}{ Cine clásico y la formación humana }} \\
\hline Sí & 82 & $10,0 \%$ & & & \\
\hline No & 0 & $0,0 \%$ & & $\mathrm{Si}$ & No \\
\hline Total & 82 & $100,0 \%$ & - Series 1 & 82 & 0 \\
\hline
\end{tabular}

Nota. Fuente: información del instrumento aplicado

Las respuestas fueron definitivas, incluso las opiniones que expresaron que no era necesario incluir el cine de Cantinflas en los contenidos del área de Humanidades estuvieron de acuerdo para afirmar que dicho género sí aporta a la formación humana; al respecto, es posible destacar algunas opiniones:

Sus películas tienen como base el servicio a la comunidad y ayudan a ponerlo en práctica durante el ejercicio de nuestra profesión, son geniales en la búsqueda de un ser humano muy humano, capaz de defender su dignidad.

Las películas, desde el contexto educativo y en la experiencia de enseñanza-aprendizaje propuesta por el docente, han invitado a la reflexión, a luchar por los ideales y forjar caminos de felicidad.

En mi caso personal, la película que vi y las que han socializado me han ayudado mejorar en el trato con los demás y a brindar mejor atención a los usuarios y clientes de la empresa.

En el aula de clase los docentes siempre enfrentan el gran reto de dar, de aportar lo mejor de sí a las generaciones del presente y el futuro, por ello buscan diferentes espacios; en tal contexto, se preguntó a los estudiantes: ¿Considera apropiada la asignatura de Humanidades para desarrollar lo pertinente al cine clásico?

Figura 7. El cine clásico en la asignatura de Humanidades

\begin{tabular}{|c|c|c|c|c|c|}
\hline Opción & Estudiantes & Porcentaje & \multirow{2}{*}{\multicolumn{3}{|c|}{ Cine clásico y la formación humana }} \\
\hline Sí & 82 & $97,6 \%$ & & & \\
\hline No & 2 & $2,4 \%$ & & & - \\
\hline Total & 82 & $100,0 \%$ & $\square$ Series 1 & $\begin{array}{c}\mathrm{Si} \\
82\end{array}$ & $\begin{array}{c}\text { No } \\
2\end{array}$ \\
\hline
\end{tabular}

Nota. Fuente: información del instrumento
Dos estudiantes insistieron en que la asignatura no es el espacio apropiado para trabajar el cine, especialmente cuando es clásico y centrado en un solo personaje; además, consideran que hay otras asignaturas pertinentes para manejar este tipo de temas, pero no especifican cuáles podrían ser o por qué no es válido el ejercicio en esta materia. Pese a ello, 97,6\% de la población estuvo de acuerdo en que la asignatura de Humanidades es apropiada para estudiar el ser humano, la formación humana y la inclusión pedagógica en el aula desde la perspectiva del cine clásico de Cantinflas; considerando que dicha estrategia permite dinamizar el proceso de enseñanzaaprendizaje y desarrollar conciencia frente a su propio papel como estudiantes y como seres humanos en la sociedad. Concretamente, afirmaron:

Sí es pertinente que se vea en la asignatura de Humanidades, ya que contribuye a fomentar los valores morales; el cine de "Cantinflas" es genial para reflexionar acerca de la vida y valorar lo que somos y tenemos. Tal vez en otra asignatura no sería tan interesante ver las películas con la perspectiva que se puede ver desde Humanidades, pues acerca al ser humano a reflexionar sobre su ser y esencia, reconociendo sus principios y valores morales.

Algunos estudiantes del proyecto ya hacen parte del ejercicio laboral en el área de la salud, los demás visualizan la realidad a la que se enfrentarán; por ello se les preguntó: ¿Pueden las películas de Mario Moreno fortalecer los principios de la formación humana de quien presta un servicio a la comunidad desde el área de la salud?

Figura 8. Películas de Mario Moreno y formación de agentes de la salud

\begin{tabular}{|c|c|c|c|c|c|}
\hline Opción & Estudiantes & Porcentaje & \multirow{3}{*}{\multicolumn{3}{|c|}{ Cine clásico y la formación humana }} \\
\hline Sí & 82 & $100 \%$ & & & \\
\hline No & 0 & $0,0 \%$ & & & \\
\hline \multirow[b]{2}{*}{ Total } & \multirow[b]{2}{*}{82} & \multirow[b]{2}{*}{$100,0 \%$} & & Si & No \\
\hline & & & $\square$ Series 1 & 82 & 2 \\
\hline
\end{tabular}

Nota. Fuente: información del instrumento aplicado

La totalidad de los estudiantes encuestados afirmó que las películas de Cantinflas fortalecen la formación humana que fundamenta el ejercicio como prestador de servicios de salud, llegando a conclusiones como que, en dicha área, es necesario ofrecer y recibir calidad, erradicando la negligencia hasta sustituirla por solidaridad, diligencia y respeto, llegando finalmente a la eficiencia. En tal sentido, expresaron:

En cada una de las películas sobresalen valores que es bueno que toda persona que trabaja en el área de la salud pueda poseer 
como riqueza inagotable, tales como la amabilidad, la humildad a la hora de atender a alguien, y el respeto como principio vital de subsistencia en la sociedad.

En casi todas las películas se evidencia la entrega, el amor y el sentido de pertenencia que se han de tener por el trabajo.

Cada película contribuye con un grano de arena para la construcción y formación de una persona integral, que sirva a la sociedad y busque el desarrollo social, preservando la dignidad del ser humano.

Las películas de Cantinflas fortalecen la formación humana, acompañan a quienes se desempeñan en el área de la salud, al llamar la atención sobre el trato a los demás; en ellas sobresale la importancia de cultivar valores como el servicio, la humildad, la colaboración y el respeto, esenciales para que quienes trabajan con la salud no vean al paciente como un negocio, con un valor económico, sino como un ser humano que requiere de la atención pertinente desde una visión que le dignifique, sin sufrir ningún tipo de exclusión.

Cada programa académico universitario cuenta con una misión, visión y un perfil para los futuros profesionales y el desempeño ocupacional; los estudiantes encuestados conocen los suyos, por ello se preguntó: ¿Puede el cine clásico, y las películas de Mario Moreno, fortalecer el perfil profesional y ocupacional del tecnólogo?

Figura 9. El cine de Mario Moreno y el perfil profesional

\begin{tabular}{|c|c|c|c|c|c|}
\hline Opción & Estudiantes & Porcentaje & \multirow{2}{*}{\multicolumn{3}{|c|}{ Cine clásico y la formación humana }} \\
\hline Sí & 82 & $100 \%$ & & & \\
\hline No & 0 & $0,0 \%$ & & & $-\infty$ \\
\hline \multirow[b]{2}{*}{ Total } & \multirow[b]{2}{*}{82} & \multirow[b]{2}{*}{$100,0 \%$} & & $\mathrm{Si}$ & No \\
\hline & & & $\square$ Series 1 & 82 & 2 \\
\hline
\end{tabular}

Nota. Fuente: información del instrumento aplicado

La respuesta es contundente y resulta de confrontar el perfil profesional-ocupacional y las películas de Cantinflas; los estudiantes afirman que el cine les ayuda a ser tecnólogos idóneos y personas de bien y con valores:

Las películas ofrecen el mensaje de que si nos comportamos como personas capaces de respetar la dignidad del ser humano, nos alejamos de la sociedad materialista en donde solo importa el poder, el tener y el placer; y pasamos a promover la esencia del ser humano integral, que apoya la inclusión de todos, es respetuoso de la diversidad y no pierde las buenas costumbres, la autenticidad y su humanidad.
Las películas expresan la importancia de la superación personal, el amor por la carrera que se está cursando y el valor de contar siempre con conocimientos actualizados, siempre siendo buenas personas; ese es el caso del Señor Doctor y de las demás películas, que dan claros ejemplos de que el ser humano puede superarse con respeto y dignidad por sí mismo. Pues todas las profesiones requieren saberes, pero es más importante saber ser persona con valores, así se podrá tener un punto de equilibrio entre estas dos realidades que desembocan en el ser mejores ciudadanos, con espíritu fuerte y bases sólidas para salir adelante.

Todas las películas me dejaron una gran enseñanza para la vida: la de luchar por ser mejor persona cada día sin pisotear la dignidad de nadie; también la necesidad de ser feliz con lo poco que se tiene y con lo que se hace, esto se refleja en cada película. Amar lo que somos y tenemos, también a quienes nos rodean, de esta manera la paz comenzará por casa, y donde hay paz habrá una felicidad que no excluye a nadie y que brilla para todas las personas sin distinción social.

A continuación se exponen los perfiles ocupacional y profesional de Regente de Farmacia y de Gestor en la Salud, con el fin de estrechar la relación entre ellos alrededor de la formación humana, destacando que es indispensable para el desarrollo social, mucho más cuando se refiere a profesionales en el área de la salud.

Figura 10. Las películas de Cantinflas y la inclusión

\begin{tabular}{|c|c|c|c|c|c|}
\hline Opción & Estudiantes & Porcentaje & \multirow{3}{*}{\multicolumn{3}{|c|}{ Cine clásico y la formación humana }} \\
\hline Sí & 82 & $100 \%$ & & & \\
\hline No & 0 & $0,0 \%$ & & & \\
\hline \multirow[b]{2}{*}{ Total } & \multirow[b]{2}{*}{82} & \multirow[b]{2}{*}{$100,0 \%$} & & $\mathrm{Si}$ & No \\
\hline & & & $\square$ Series 1 & 82 & 2 \\
\hline
\end{tabular}

Nota. Fuente: información del instrumento aplicado

La investigación pregunta por la relación entre las películas de Mario Moreno y la inclusión o exclusión que se presenta en ellas; todos los estudiantes afirmaron que ninguna película presenta situaciones de exclusión. Se expresaron de la siguiente forma:

En todo momento el personaje principal combate la injusticia, evita que un gamonal del pueblo se aproveche de los pobres y los explote por el deseo del tener más. Se enfrenta a jefes orgullosos que humillan a sus empleados por diferentes razones y a quienes creen que por tener o saber algo más se aprovechan de los demás, llevándolos a la exclusión de la sociedad por miedo a la represión, la burla y otros aspectos que denigran y deshumanizan al hombre.

De las películas que se han visto, y de aquellas que han socializado los compañeros, se pueden evidenciar signos de exclusión hacia 
una persona de manera radical, pues hay intentos, como en las películas El Profe o El Padrecito, en donde se quiere expulsar de la plaza pública a quien intenta sacar a la gente de la ignorancia, a los que buscan la felicidad de los demás. Es evidente la lucha por superar los brotes de exclusión social que se presentan, recuperando la dignidad del ser humano para incluir a los demás en la sociedad.

El trabajo también incluyó una pregunta por la relación entre las películas de Cantinflas y la interculturalidad; el fenómeno resulta importante en la medida en que existe un proceso de comunicación e interacción entre diferentes grupos sociales y Cantinflas quien, no permite que nadie esté por encima del otro, favoreciendo permanentemente la integración social y la convivencia. La Figura 11 da cuenta de las respuestas.

Figura 11. El cine clásico y la interculturalidad

\begin{tabular}{|c|c|c|c|c|c|}
\hline Opción & Estudiantes & Porcentaje & \multirow{2}{*}{\multicolumn{3}{|c|}{ Cine clásico y la formación humana }} \\
\hline Sí & 82 & $100 \%$ & & & \\
\hline No & 0 & $0,0 \%$ & & & $-\infty$ \\
\hline \multirow[b]{2}{*}{ Total } & \multirow[b]{2}{*}{82} & \multirow[b]{2}{*}{$100,0 \%$} & & SI & No \\
\hline & & & Series 1 & 82 & 2 \\
\hline
\end{tabular}

Nota. Fuente: información del instrumento aplicado

Para los estudiantes, el cine de Mario Moreno presenta una tensión por mantener el punto de equilibrio entre los distintos espacios, buscando acuerdos hasta llegar a la armonía social. Se da una lucha por el respeto a cada persona sin importar su condición religiosa, política o racial, evidenciando un apoyo irrestricto a la interculturalidad que favorece la autenticidad y lo propio de cada cultura, valorando las raíces y la tradición.

Por otra parte, la investigación se ocupa de la pedagogía en la enseñanza-aprendizaje, por lo cual ve la didáctica como un elemento dinamizador del proceso que da claridad visual y estratégica; por ello, también se preguntó: ¿Cuál organizador gráfico eligió usted para dar a conocer a sus compañeros la película que trabajó?

Figura 12. Organizadores gráficos

\begin{tabular}{|c|c|c|c|c|c|c|c|}
\hline Opción & Estudiantes & Porcentaje & \multirow{2}{*}{\multicolumn{5}{|c|}{ Cine clásico y la formación humana }} \\
\hline a. Mapa conceptual & 15 & $18,3 \%$ & & & & & \\
\hline b. Mapa mental & 14 & $17,1 \%$ & & \multirow{2}{*}{\multicolumn{4}{|c|}{57 E1 }} \\
\hline c. Sopa de letras & 22 & $26,8 \%$ & & & & & \\
\hline d. Otros & 31 & $37,8 \%$ & & 1 & 2 & 3 & 4 \\
\hline Total & 82 & $100,0 \%$ & $\square$ Series 1 & 15 & 14 & 22 & 31 \\
\hline
\end{tabular}

Nota. Fuente: información del instrumento aplicado
El organizador gráfico es una opción didáctica para construir aprendizaje significativo y facilita al docente la enseñanza proactiva. La representación gráfica permite la aprehensión y asimilación de saberes, contribuyendo a un proceso educativo dinámico, productivo y activo para docentes y estudiantes. Frente a la pregunta que comprendía al organizador gráfico, el mayor porcentaje de estudiantes se identificó con la opción "otro", y el menor con la de "mapa metal"; es interesante la inclinación de los alumnos por la sopa de letras, pues sus ideas van más allá, planteando acrósticos, videos, dibujos y crucigramas, entre otros que exponen su creatividad a la hora de dar a conocer gráficamente la película que vieron y de paso socializarla.

La variedad de películas de Mario Moreno permitió que los estudiantes eligieran una de ellas para luego identificar distintos elementos, como personajes principales, secundarios, contextos, entre otros; aspectos de fondo, como la formación humana y valores; y aspectos como la inclusión e interculturalidad. Frente a ello se preguntó: ¿Cuál de las películas de Mario Moreno eligió para profundizar en la formación humana? A continuación se presenta el listado de películas y la cantidad de estudiantes que las vieron.

Tabla 3. Listado de películas

\begin{tabular}{|c|c|c|}
\hline$N^{\circ}$. & Película & Número de estudiantes \\
\hline 1 & El barrendero (1981) & 1111 \\
\hline 2 & El patrullero 777 (1977) & II \\
\hline 3 & El ministro y yo (1975) & III \\
\hline 4 & Don Quijote cabalga de nuevo (1972) & III \\
\hline 5 & El profe $(1970)$ & 11111 \\
\hline 6 & Un Quijote sin mancha (1969) & 111 \\
\hline 7 & Por mis pistolas (1968) & II \\
\hline 8 & Su excelencia (1966) & 11111 \\
\hline 9 & El señor doctor (1965) & 1111111111 \\
\hline 10 & El padrecito (1964) & 1111111 \\
\hline 11 & El analfabeto (1960) & 11111111111111111 \\
\hline 12 & El super sabio (1948) & III \\
\hline 13 & El circo (1963) & III \\
\hline 14 & A volar joven (1947) & III \\
\hline 15 & Romeo y Julieta ( ...) & III \\
\hline 16 & El mago $(\ldots)$ & 111 \\
\hline 17 & Los tres mosqueteros ( ...) & II \\
\hline 18 & Un día con el diablo ( ...) & II \\
\hline 19 & Si yo fuera diputado ( ...) & II \\
\hline
\end{tabular}

Durante el proyecto, como se trata de un proceso de aula, se buscó descubrir aspectos como la formación humana, los valores 
más significativos y la forma en que se expresan desde las cintas para la vida como tecnólogos; por ello, a la hora de socializar las experiencias, se preguntó: ¿Cuál fue la película que más le impactó? Este fue uno de los instantes más reveladores, pues dio lugar a las disertaciones orales, espacios de desarrollo social, de integración y de compartir como personas. Cada alumno tuvo tiempo y espacio para socializar la película que vio, y de realizar las diferentes actividades que le permitieron aprehender su experiencia; la película que impactó su vida no fue necesariamente la misma que escogió, pues algunos de ellos fueron tocados por la exposición de sus compañeros.

El trabajo desarrollado también requería preguntar a los estudiantes por sus reflexiones sobre los personajes principales y las escenas de la película que trabajaron; en términos generales, sus respuestas apuntaron a señalar que los personajes principales eran personas sencillas, humanas ante todo, preocupadas por su entorno y con situaciones de vida muy fuertes, incluso inhumanas. Allí entra el reto de Cantinflas: la humanización; desde una invitación para adaptarse y desenvolverse en cualquier situación o cultura. Las películas se desarrollan entre tensiones fuertes, con personas humildes, de gran corazón, y otras posesivas, que creen poder dominar a la sociedad y solo les importa el poder.

Evidentemente, los estudiantes señalaron a Cantinflas como el mejor de los personajes principales, una persona humilde, perseverante, humana y con mucho amor para dar; para ellos, su comportamiento se relaciona con el viejo refrán de: Haz el bien y no mires a quien. Sin importar el oficio que desempeñe, enfrenta cada situación de la vida evitando la exclusión, la discriminación, mientras favorece el respeto por la dignidad humana. Aunque algunos personajes principales actuaban como antagonistas, Cantinflas se enfocaba en dar a entender la importancia de favorecer la dignidad humana, los valores de cada persona ante la sociedad; desde allí, surge la lucha por lo que se quiere, porque en la vida hay que hacer posible lo imposible con sacrificios, fortaleciendo a personas que encarnen el deseo de felicidad, de hacer el bien.

Finalmente, la experiencia de ver películas durante la asignatura de Humanidades permitió afianzar en los estudiantes la idea de que es importante consolidar la formación humana dentro del perfil profesional y ocupacional; sin embargo, para comprobarlo se preguntó: ¿Qué enseñanza le queda, para su vida y formación humana, del trabajo realizado desde el cine clásico y las películas de Mario Moreno? Las respuestas de los estudiantes fueron positivas y dieron cuenta de múltiples enseñanzas personales obtenidas gracias a su experiencia con las películas, las cuales abarcaron su vida personal, familiar y laboral, hasta concluir que la formación humana es indispensable para la sociedad:

En mi opinión, la película de El Analfabeto nos da una reflexión sobre no aprovecharnos de las personas que no saben, sino que, por el contrario, hay que ayudarlas. La mayor de las enseñanzas es que debemos prepararnos para la vida, pero no olvidar los valores que nos hacen verdaderas personas. Se trata de colocarnos en los zapatos de los otros para poderlos entender, comprender y ayudar; lo importante es constituirnos como seres humanos integrales, sin dejarnos influenciar por las condiciones de prejuicio.

Con sus películas, Mario Moreno se constituye en un educador de valores, da el ejemplo de que como personas tenemos errores pero que se pueden corregir con la buena voluntad. Es necesario mantener los principios de buena convivencia en el trabajo, en el hogar y en todo lugar de la sociedad; la enseñanza más importante es la de valores como el servicio, la honestidad, la humildad y la perseverancia, que nos llevan a constituirnos como verdaderas personas. Con Cantinflas se entiende que pueden existir problemas en la vida, pero hay que buscarles el lado bueno y amable si se quiere encontrar una posible solución; no podemos decaer en nuestros sueños, debemos convertir los obstáculos en fortalezas y fortificar nuestra formación humana, para aprovechar las oportunidades.

\section{Conclusiones y recomendaciones}

La presente investigación pedagógica nace del trabajo en el aula de clase e involucró la intervención de los actores del acto educativo, estudiantes y docentes, desde la asignatura de Humanidades, consolidando el desarrollo curricular. La experiencia fue un mecanismo para aprender sobre la importancia de fortalecer la formación humana, los principios de inclusión e interculturalidad, en medio de un mundo globalizado, desde las películas de Cantinflas. El ejercicio se constituyó en un trabajo académico que dio frutos para el desarrollo de los contenidos de la asignatura de Humanidades.

El análisis de cada película permitió a los estudiantes encontrar situaciones que les resultan vitales para la formación en valores $\mathrm{y}$, en ese sentido, la experiencia pedagógica arrojó resultados positivos que se hicieron evidentes en la socialización; las actividades fortalecieron el vínculo entre el alumno, la carrera y la sociedad, al punto de que no giraron alrededor de una calificación, sino de la sensibilidad que despertó en las vidas de los actores. 
TEMA: INDUSTRIAS CULTURALES Y EDUCACIÓN

\section{Referencias}

Argüelles P., D. (2010, Julio-Diciembre). Estrategias para promover procesos de aprendizaje autónomo. Revista Escuela de Administración de Negocios (EAN), No. 69, pp. 209-211.

Castillo, S. M. (2004). Guía para la formulación de proyectos de investigación. Bogotá: Magisterio.

Echeverry, A. J. (1999, Enero-Junio). La historia en la formación humana. Revista Gestión y Desarrollo, No. 3, pp. 53-60.

Forbes, O., et al. (1992). Cómo entendemos la interculturalidad. Revista de Educación y cultura, No. 27, pp. 17-19.

García, C. N. (1999). La globalización y la interculturalidad narrada por los antropólogos. Revista del departamento de Antropología, No. 14, pp. 19-41.

González, M. I. (2005). La escuela: espacio de reconocimiento de la interculturalidad. Revista Pedagogía y Saberes, No. 22, pp. 49-56.

Gutiérrez, C. (1991). La investigación en Colombia en las artes, las humanidades y las ciencias sociales. Bogotá: Uniandes.

Hernández, S. R., et al. (2010). Metodología de la investigación. Bogotá: McGraw Hill.

Munevar, D. (2008). Articulaciones educativas para la inclusión de la diversidad. Revista Colombiana de Educación, pp. 36-57.

Nussbaum, M. (2010). Sin fines de lucro. Por qué la democracia necesita de las Humanidades. Madrid: Katz.

Recio, A. (1998-Mayo). La formación humana y social en el área de Ciencias de la Educación. Itinerario Educativo, pp. 53-64. 